\title{
An Integrated Communication Method for LED Intelligent Dimming System with Switching Ripple Communication
}

\author{
Wenyang Shang \\ School of Electrical and Automation \\ Engineering \\ Nanjing Normal University \\ Nanjing, China \\ 1982194306@qq.com
}

Fulong Li

School of Mechanical, Electrical and

Manufacturing Engineering

Loughborough University

Leicestershire, UK

F.Li@lboro.ac.uk

\author{
Ruichi Wang \\ School of Electrical and Automation \\ Engineering \\ Nanjing Normal University \\ Nanjing, China \\ richwang@njnu.edu.cn
}

Jiande $\mathrm{Wu}$

College of Electrical Engineering

Zhejiang University

Hangzhou, China

eewjd@zju.edu.cn

\author{
Zhengyu Lin \\ School of Mechanical, Electrical and \\ Manufacturing Engineering \\ Loughborough University \\ Leicestershire, UK \\ z.lin@ieee.org
}

\begin{abstract}
Aiming at solving the problems of complex wiring and high cost of the existing distributed light emitting diode (LED) dimming system, a new LED intelligent dimming system is proposed in this paper. Power/signal multiplexing modulation and transmission technology is applied to the LED drive circuits, and power and signal simultaneously transmission through the power bus is achieved. Quaternary differential phase shift keying (QDPSK) and orthogonal frequency division multiplexing (OFDM) are used to improve the communication efficiency. It realizes the communication between multiple LEDs for intelligent dimming control without additional hardware circuit, which greatly reduce the system volume, cost and complexity, enhancing the system reliability as well. Finally, the correctness and feasibility of the LED intelligent dimming strategy is verified by PSIM simulations.
\end{abstract}

Keywords-dc-dc converter, switching ripple communication, differential phase shift keying (DPSK), LED intelligent dimming

\section{INTRODUCTION}

With the rapid development of global urbanization, it promotes the rapid construction of smart cities, of which intelligent light emitting diode (LED) lighting system is one of the key technologies ${ }^{[1]}$. Compared with traditional incandescent lamps and fluorescent lamps, LED is widely used in various lighting applications for its high brightness, small volume, long life and rich color ${ }^{[2]}$. Consequently, the control of LED lighting system is particularly important and attracts a lot of attention ${ }^{[3],[4]}$.

In LED lighting systems, LED drive circuits usually adopt current control strategy and pulse width modulation (PWM) mode is used to adjust brightness ${ }^{[5]-[7]}$. For distributed lighting system with a large number of LEDs, real-time communication between LEDs or between LEDs and the host computer is indispensable for reliable and intelligent control. In conventional solutions, field bus communication is commonly used. Literatures [8] and [9] propose an LED remote lighting control system based on controller area network (CAN) bus communication. Compared with manual adjustment method, it realizes intelligent control, but do not consider the use of sunlight in indoor LED lighting, which may lead to excessive lighting. In [10], CAN bus is used as the medium of communication with sensors and actuators to control the LED light intensity to a satisfactory level with the least energy consumption. Field-bus based communication has the advantages of simple and mature, but it needs additional communication wires and data transmitters, which increase the system cost and volume, and decrease the system reliability.

In order to simplify the connection of the system, two improved solutions can be adopted, which are wireless communication and power line communication (PLC). ZigBee represented wireless communication method has been widely applied to road lighting ${ }^{[1],[11]-[13]}$, office lighting ${ }^{[14]}$ and home lighting ${ }^{[15]-[17]}$. In [11], using ZigBee communication technology, a remote-control system of street lamp powered by solar energy is designed. The lighting is controlled by the central management station, and the adjustment is not realtime. In [18], an intelligent street lamp lighting system based on traffic flow is proposed, which can adjust the brightness of street lamps in real-time according to the traffic flow, so as to reduce energy consumption. In [1], the intelligent LED street lamp system integrates the function of public meteorological data perception, which can reduce traffic accidents caused by low visibility. Although wireless networks are more and more widely used, with the reduction of radio frequency (RF) spectrum, short-range communication technologies such as wireless local area network (WLAN) and ZigBee are causing bandwidth disputes between adjacent devices at the same time ${ }^{[19]}$. In addition, RF systems are usually subject to electromagnetic interference, and microwave can cause harm to human body. Therefore, the security and reliability of wireless networks are often questioned ${ }^{[20]}$.

PLC technology multiplexes power lines of various voltage levels for data transmission. The extensive use of existing power lines can significantly reduce the wiring of the system, and enhance the reliability ${ }^{[21]-[23]}$. In [22], half duplex communication with PLC technology is used to control the LED lighting system. Compared with Ethernet, there is no complex communication protocol, but the communication efficiency is low. In [23], a LED lighting control system based on P-BUS is designed, which uses fewer communication lines and components, and has strong anti-interference ability and high communication efficiency. However, PLC needs to arrange additional signal coupling circuit to inject the data to the power line, and amplify the communication signal through 
independent amplifier circuit, which increases the volume and cost of the system.

LED drive circuits usually adopt power electronic converters, thus switching ripples inevitably occur at the input and output ports. If the ripple can be modulated, power conversion and data communication can be realized simultaneously, which is the basic idea of switching ripple communication $^{[24]}$. It does not need additional signal transmission circuit, which greatly simplifies the hardware circuit.

This paper is arranged as follows: Section II presents the basic architecture of the LED intelligent dimming control system, Section III illustrates the principle of the system. In addition, the quaternary differential phase shift keying (QDPSK) modulation process and the corresponding demodulation strategy are presented. In Section IV, orthogonal frequency division multiplexing (OFDM) strategy for multi-machine communication is presented. In Section V, simulation results based on PSIM are presented to verify the correctness of the scheme. Finally, conclusions are made in Section VI.

\section{LED INTELLIGENT DIMMING CONTROL SYSTEM ARCHITECTURE}

The block diagram of LED intelligent dimming control system with switching ripple communication is shown in Fig. 1 , including direct current (DC) power supply, LED drive circuits, an upper computer and LED load. Among them, LED drive circuits adopt boost converter and constant current control is adopted.. In this system, multiple LED drive circuits are connected in parallel to the DC bus, and are powered by a DC power supply. Each converter is multiplexed as a data transmitter, correspondingly, the common DC bus is multiplexed as communication channel. The system can select a host computer to communicate with LED drive circuits, and then control the LED lighting system. It can also communicate with each other directly between LED drive circuits without the need of host computer to control different dimming, which improves the control efficiency of dimming.

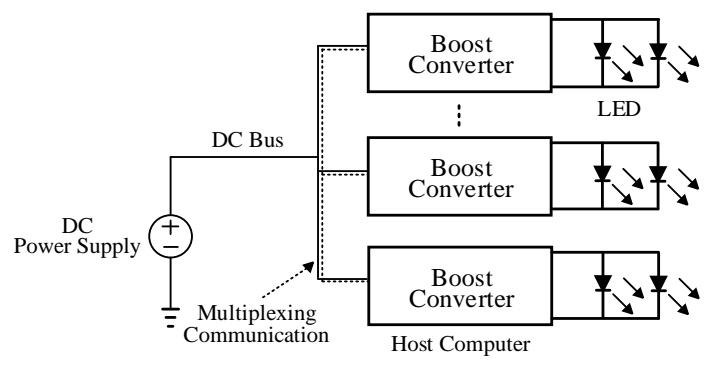

Fig. 1. Block diagram of LED lighting control system.

\section{RIPPLE COMMUNICATION DESIGN}

Taking power PWM/data PSK multiplexing modulation as an example, this section addresses the basic principle of switching ripple communication. Besides, this section also analyzes the QDPSK modulation process in detail, and gives the corresponding demodulation strategy.

\section{A. Basic Principle}

In the process of power conversion, the output DC compo-

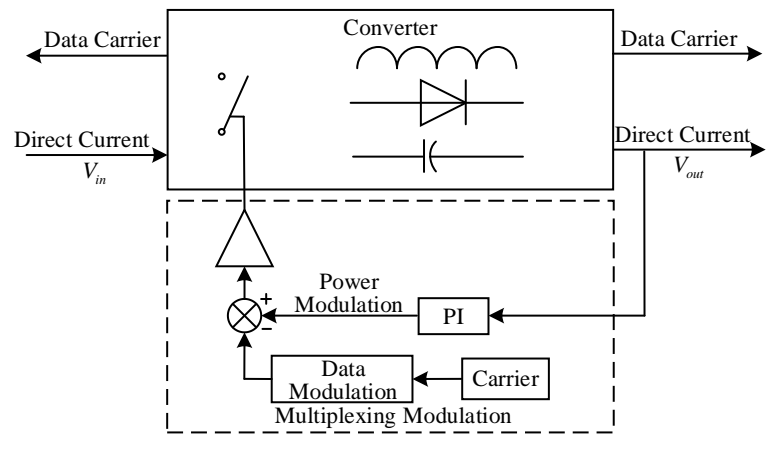

Fig. 2. Power/signal multiplexing modulation principle block diagram.

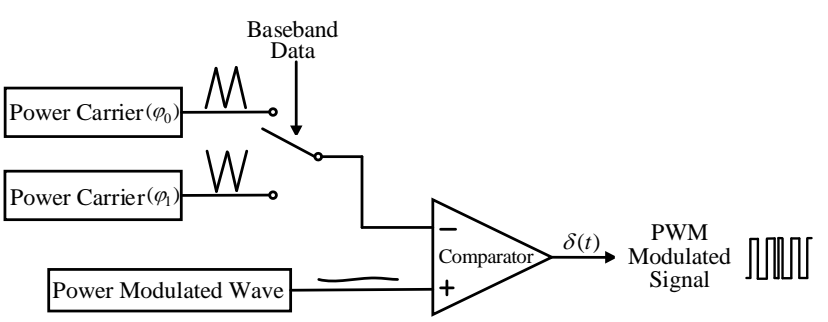

Fig. 3. Principle block diagram of power PWM/data PSK modulation.

nent is controlled by adjusting the duty cycle of PWM sequence, which is independent of its frequency and phase. Although filtered, the switching ripple is still inevitable at the input and output ports of the converter. Therefore, if the frequency and phase of the switching ripple can be modulated while the duty cycle is left constant, the power/signal multiplexing modulation and transmission can be achieved.

The basic idea of power/signal multiplexing modulation and transmission is to modulate the frequency or phase of power carrier while adjusting the duty cycle of power carrier to control the stable DC output. There are two basic modulation methods for power/signal multiplexing modulation: carrier frequency shift keying modulation and carrier phase shift keying modulation. This paper only studies the carrier phase shift keying modulation system for its lower bit error rate and strong anti-interference ability. The principle block diagram of power/signal multiplexing modulation of carrier PSK modulation is shown in Fig. 2. In PSK modulation, the definitions of data signal " 0 " and " 1 " are as follows: the phase $\varphi_{0}$ of power carrier represents data " 0 ", and the phase $\varphi_{1}$ represents data "1". When there is no signal transmitted, the phase of power carrier is also kept $\varphi_{0}$. The modulation process is shown in Fig. 3, and after PSK modulation, the data carrier is used as power PWM carrier of switching converter.

Fig. 4 shows the block diagram of power/signal multiplexing modulation of LED lighting system. The converter adopts current closed-loop control to provide power with constant current for LED. The boost converter is multiplexed as a signal generation circuit. On the one hand, it controls the brightness of LED by adjusting the PWM duty cycle; on the other hand, it modulates the data by adjusting the PWM power carrier, so that the switch ripple carries the data signal, based on which intelligent dimming control is realized. The modulated data signal is transmitted on the power line, so there is no need to arrange additional communication wires. 


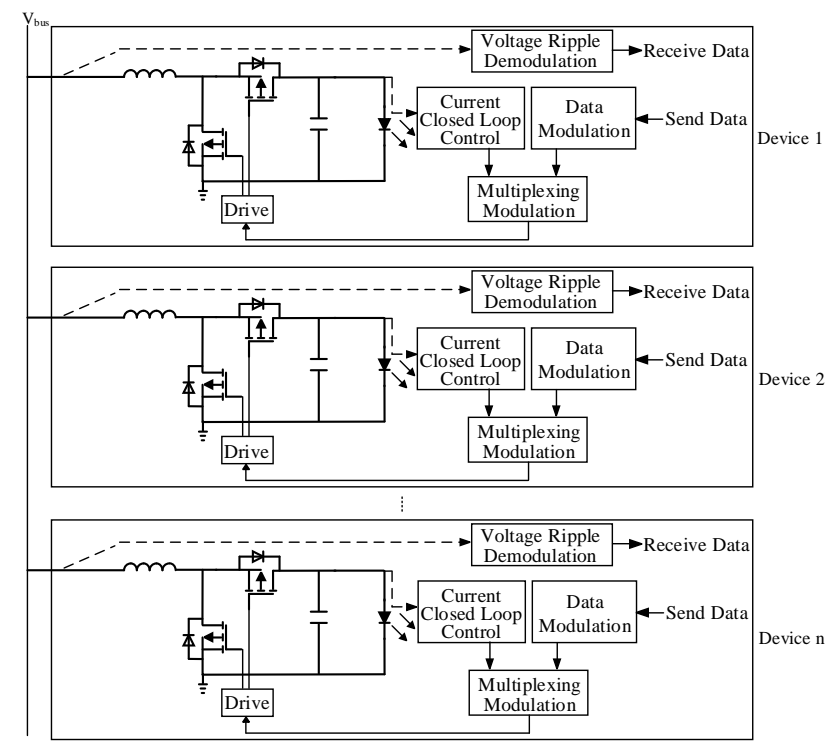

Fig. 4. Power/signal multiplexing modulation block diagram of LED lighting system.

TABLE I. RELATIONSHIP BETWEEN DoubLE BIT SYMBOL AND CARRIER PHASE CHANGE

\begin{tabular}{|c|c|c|c|}
\hline \multirow{2}{*}{ Double Bit Symbol } & \multicolumn{2}{c|}{ Carrier Relative Phase Change } \\
\cline { 3 - 4 } & & Mode A & Mode B \\
\hline 0 & 0 & $0^{\circ}$ & $225^{\circ}$ \\
\hline 0 & 1 & $90^{\circ}$ & $135^{\circ}$ \\
\hline 1 & 1 & $180^{\circ}$ & $45^{\circ}$ \\
\hline 1 & 0 & $270^{\circ}$ & $315^{\circ}$ \\
\hline
\end{tabular}

To increase the communication rate, quaternary phase shift keying (QPSK) modulation is adopted in this paper. It uses four different phase carriers to represent digital information. In the process of signal coherent demodulation, the ambiguity of $0, \pi, 2 / \pi, 3 \pi / 2$ of local carrier phase will lead to the phenomenon of phase ambiguity in demodulation. So, in practical application, quaternary differential phase shift keying (QDPSK) modulation is usually used.

QDPSK is a kind of M-ary phase shift keying modulation method, its modulation principle is similar to QPSK modulation, but QDPSK signal represents digital information by recognizing the change of carrier relative phase between adjacent symbols. If the phase of the pilot double bit symbols are taken as the reference, $\Delta \varphi$ is the phase difference between the current symbol and the pilot symbol, then the coding rules of QDPSK signal are shown in Table I. QDPSK signal can be generated by quadrature phase modulation. Fig. 5 shows the principle block diagram of generating QDPSK signal according to the rule of mode $\mathrm{A}$ in Table I.

Among them, the serial/parallel conversion module transforms the binary baseband serial signal $s(t)$ into two parallel symbol $a$ and $b$. The coding transformation module transforms the parallel absolute codes $a$ and $b$ into the relative codes $c$ and $d$. According to the relevant modulation rules shown in Table I, the corresponding relationship between the pilot double bit symbols $c_{n-1}, d_{n-1}$ and the current double bit

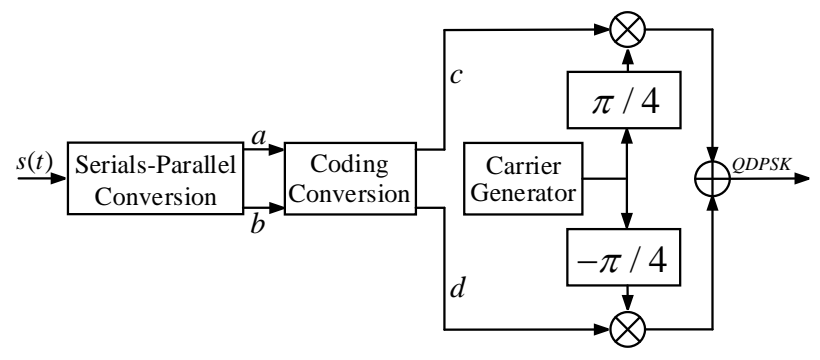

Fig. 5. Principle block diagram of QDPSK signal generation.

TABLE II. CORRESPONDING RELATIONSHIP BETWEEN SYMBOL AND PHASE

\begin{tabular}{|c|c|c|c|c|c|c|c|c|}
\hline \multicolumn{3}{|c|}{ Pilot Symbol State } & \multicolumn{3}{|c|}{$\begin{array}{c}\text { Arrived Double Bit } \\
\text { Symbol }\end{array}$} & \multicolumn{3}{|c|}{$\begin{array}{c}\text { Current Symbol } \\
\text { State }\end{array}$} \\
\hline$\varphi_{n-1}$ & $c_{n-1}$ & $d_{n-1}$ & $a_{n}$ & $b_{n}$ & $\Delta \varphi_{n}$ & $\varphi_{n}$ & $c_{n}$ & $d_{n}$ \\
\hline \multirow{4}{*}{$0^{\circ}$} & \multirow{4}{*}{0} & \multirow{4}{*}{0} & 0 & 0 & $0^{\circ}$ & $0^{\circ}$ & 0 & 0 \\
\hline & & & 0 & 1 & $90^{\circ}$ & $90^{\circ}$ & 0 & 1 \\
\hline & & & 1 & 1 & $180^{\circ}$ & $180^{\circ}$ & 1 & 1 \\
\hline & & & 1 & 0 & $270^{\circ}$ & $270^{\circ}$ & 1 & 0 \\
\hline \multirow{4}{*}{$90^{\circ}$} & \multirow{4}{*}{0} & \multirow{4}{*}{1} & 0 & 0 & $0^{\circ}$ & $90^{\circ}$ & 0 & 1 \\
\hline & & & 0 & 1 & $90^{\circ}$ & $180^{\circ}$ & 1 & 1 \\
\hline & & & 1 & 1 & $180^{\circ}$ & $270^{\circ}$ & 1 & 0 \\
\hline & & & 1 & 0 & $270^{\circ}$ & $0^{\circ}$ & 0 & 0 \\
\hline \multirow{4}{*}{$180^{\circ}$} & \multirow{4}{*}{1} & \multirow{4}{*}{1} & 0 & 0 & $0^{\circ}$ & $180^{\circ}$ & 1 & 1 \\
\hline & & & 0 & 1 & $90^{\circ}$ & $270^{\circ}$ & 1 & 0 \\
\hline & & & 1 & 1 & $180^{\circ}$ & $0^{\circ}$ & 0 & 0 \\
\hline & & & 1 & 0 & $270^{\circ}$ & $90^{\circ}$ & 0 & 1 \\
\hline \multirow{4}{*}{$270^{\circ}$} & \multirow{4}{*}{1} & \multirow{4}{*}{0} & 0 & 0 & $0^{\circ}$ & $270^{\circ}$ & 1 & 0 \\
\hline & & & 0 & 1 & $90^{\circ}$ & $0^{\circ}$ & 0 & 0 \\
\hline & & & 1 & 1 & $180^{\circ}$ & $90^{\circ}$ & 0 & 1 \\
\hline & & & 1 & 0 & $270^{\circ}$ & $180^{\circ}$ & 1 & 1 \\
\hline
\end{tabular}

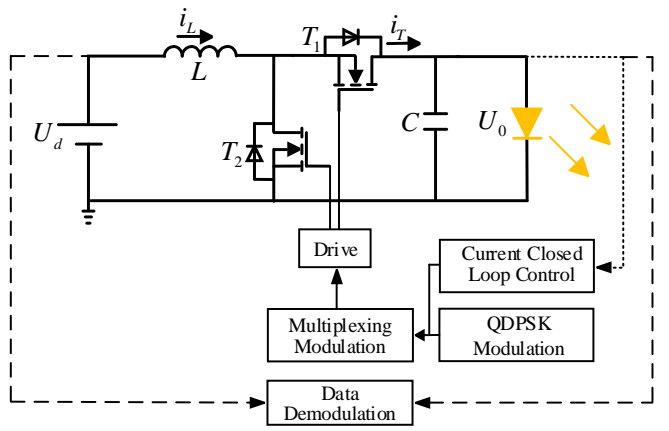

Fig. 6. Principle block diagram of power PWM/data QDPSK modulation.

symbols $c_{n}$ and $d_{n}$ under the mode A coding rule is shown in Table II. Mode B follows the same rule.

Fig. 6 shows the block diagram of power/signal multiplexing modulation with QDPSK modulation for LED drive circuit. The signal modulation part adopts QDPSK modulation, which can improve the data transmission rate of the converter.

Some key waveforms in the power/signal multiplexing modulation are shown in Fig. 7. Fig. 7(a) shows the baseband quaternary signal. Fig. 7(b) shows a quaternary data signal that the baseband signal becomes relative codes after coding transformation. Fig. 7(c) shows the carrier modulated by 


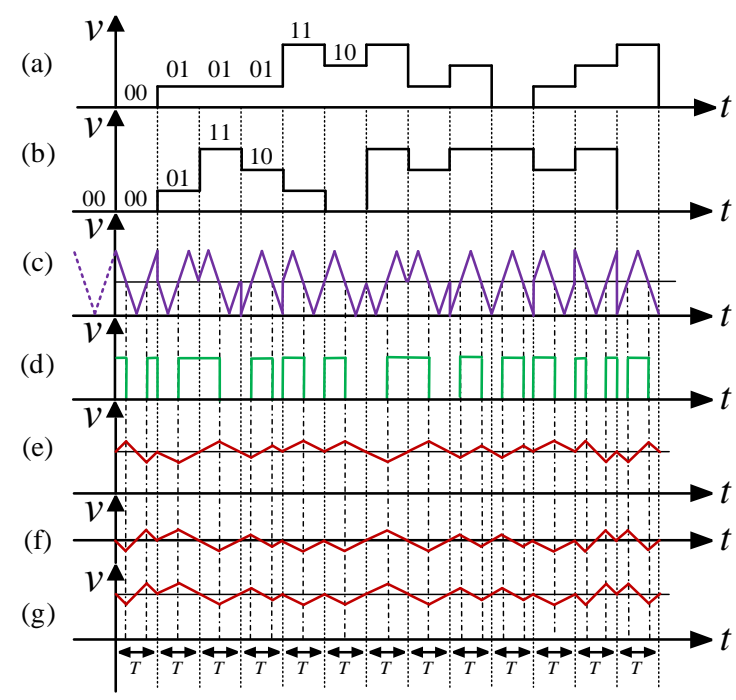

Fig. 7. Key waveforms of boost circuit based on QDPSK multiplexing modulation.

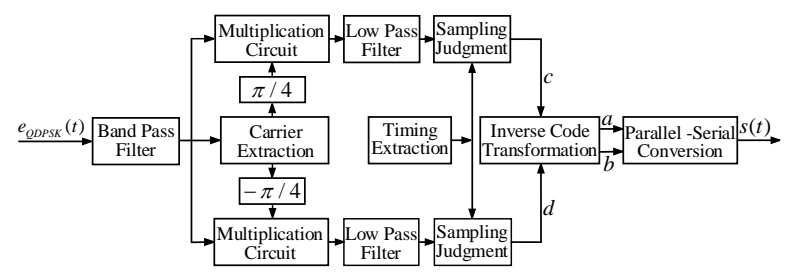

Fig. 8. Demodulation principle block diagram of QDPSK signal coherent demodulation method.

QDPSK. After the triangle power carrier and the power reference signal pass through the voltage comparator, it generates the gate control signal driving the power electronic circuit converter switch action, as shown in Fig. 7(d). Fig. 7 (e) shows the inductance current waveform of boost converter. Fig. 7(f) shows the output current ripple waveform of boost converter; The output voltage waveform is shown in Fig. $7(\mathrm{~g})$.

From Fig. 7(c) and Fig. 7(d), it can be seen that there are four different phases on the power carrier and the gate signal of switch tube, and from Fig. 7(e) to Fig. 7(g), four different phases are found on the input and output current ripple of the boost converter. This shows that the PWM triangle carrier, as well as the input and output port of the converter can carry the quaternary signal after QDPSK modulation.

\section{B. Demodulation Strategy}

After QDPSK modulation of PWM power carrier of converter in LED drive circuits, data signal will be transmitted to ripple signal of input and output of converter, and coherent demodulation method can be used to demodulate ripple signal. The principle of coherent demodulation of QDPSK signal is similar to that of QPSK signal, except that a step of inverse coding transformation is added in the later part to restore relative codes to absolute codes. The demodulation principle block diagram of QDPSK signal coherent demodulation method under mode A coding rule is shown in Fig. 8. The QDPSK signal passing through the band-pass filter can be

regarded as the signal superimposed by two sinusoidal signals, which can be recovered by coherent demodulation.

\section{RESEARCH ON MULTI-MACHINE COMMUNICATION}

\section{A. OFDM Strategy}

As shown in Fig. 1, the distributed LED lighting system contains a large number of LEDs. Multiple LEDs may need to communicate with each other at the same, which will cause inter channel interference and increase the difficulty of signal demodulation. As a kind of multi-carrier parallel-transmission modulation technology, OFDM technology divides the channel into several sub-channels, and the original data is modulated to the orthogonal carriers (sub-channels) respectively, so as to realize multi-channel parallel transmission. It not only improves the system communication efficiency, but also solve the problem of inter symbol crosstalk. At the receiver, although the spectrum of each subcarrier is partially overlapped, because the sub-carriers on the sub-channel are strictly orthogonal, it is still able to distinguish each sub-carrier and realize signal demodulation.

Fig. 9 shows the modulation principle block diagram of OFDM technology. The information sequence to be transmitted is divided into $\mathrm{N}$-channel sub-sequences, and the symbol rate of each channel is reduced by $1 / \mathrm{N}$ times. $\mathrm{N}$ channel carriers are modulated by these sub-sequences respectively to realize the parallel transmission of data signals. If the bit rate of each channel is kept unchanged, the total information transmission rate will be increased by $\mathrm{N}$ times.

In OFDM system, the channel is divided into $\mathrm{N}$ subchannels, and the sub-carriers on each sub-channel is

$$
x_{k}(t)=A_{k} \cos \left(2 \pi f_{k} t+\varphi_{k}\right) \quad k=0,1,2, \cdots, N-1
$$

Where $x_{k}(t)$ is the k-th sub-carrier and $A_{k}$ is the amplitude of the sub-carrier; $f_{k}$ is the frequency of the sub-carrier; $\varphi_{k}$ is the phase of the sub-carrier. Therefore, n-channel subsignals in OFDM system can be expressed as

$$
s(t)=\sum_{k=0}^{N-1} x_{k}(t)=\sum_{k=0}^{N-1} A_{k} \cos \left(2 \pi f_{k} t+\varphi_{k}\right)
$$

In order to completely separate these N-channel subsignals at the receiving end, they are required to be orthogonal in each symbol period:

$$
\int_{0}^{T_{s}} \cos \left(2 \pi f_{k} t+\varphi_{k}\right) \cos \left(2 \pi f_{i} t+\varphi_{i}\right) d t=0
$$

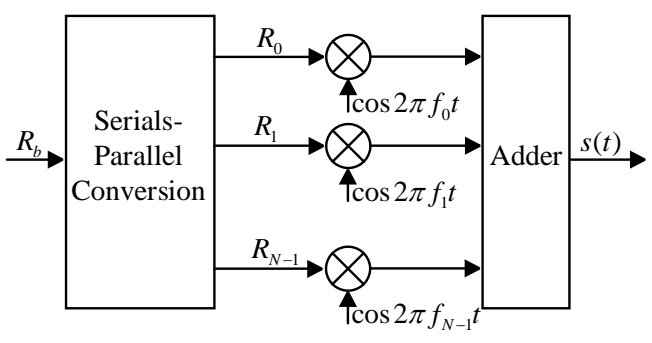

Fig. 9. OFDM modulation principle diagram. 


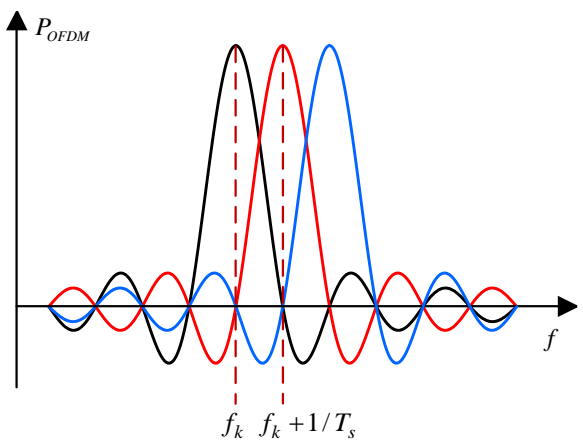

Fig. 10. Multi-channel sub-carriers synthesis spectrum.

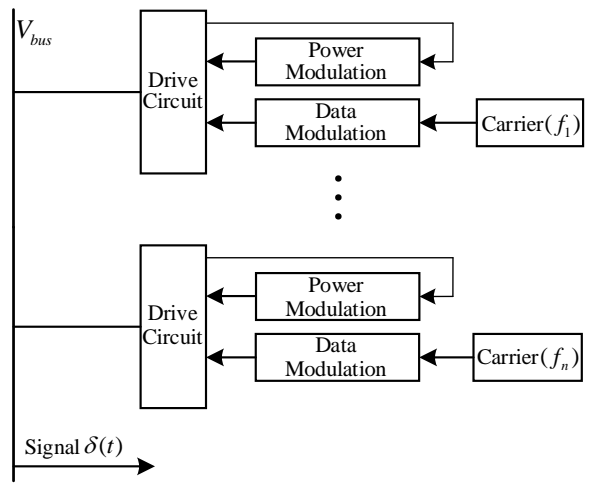

Fig. 11. Principle block diagram of OFDM power/signal multiplexing modulation.

After simplification by trigonometric formula, it can be deduced that when the subcarrier frequency of each channel meets the constraint:

$$
f_{k}=\frac{k}{2 T_{S}} \quad k=0,1,2, \cdots, N-1
$$

The sub-signals can be orthogonal to each other. And the carrier frequency interval of any two channels meets the requirement

$$
\Delta f=f_{k}-f_{i}=\frac{n}{T_{S}}
$$

That $\mathrm{n}$ is an integer. When it is taken as 1 , it can maximize the utilization of the frequency band. Therefore, the frequency interval of the minimum subcarrier is

$$
\Delta f_{\min }=\frac{1}{T_{S}}
$$

In the frequency domain, although the multi-channel subcarriers which meet the above constraint are partially overlapped in the frequency spectrum after being synthesized by OFDM technology, as shown in Fig. 10, they are still orthogonal in each symbol time, so they can be completely distinguished in the receiver demodulation.

Using QDPSK and OFDM technology for LED drive circuits can not only realize multi-machine communication between LEDs, but also improve the information transmission rate of multiplexing communication. Fig. 11 shows the block diagram of power/signal multiplexing modulation principle using OFDM technology. In the diagram, the LED drive circuits are powered by the common DC bus, which perform power/signal multiplexing modulation on its switching ripple, and receive data signals from the DC bus, thus realizing the function of transmitting control signals on the power line.

\section{B. Noise Analysis}

Headings, or heads, are organizational devices that guide the reader through your paper. There are two types: component heads and text heads.

Noise always exists in the communication system, which is a kind of interference in the channel, so noise analysis is very important to the communication system. In this dimming system, because the signal is transmitted through the power line, power noises are the main components, including switching noise, PI adjustment process noise and so on. The power supply bus voltage is DC voltage, which can be filtered easily after filtering, regardless of its impact on the communication system.

The main noise caused by switching action of converter is fundamental wave, high order harmonic and its sideband components, and voltage spike caused by switching action. Due to the high frequency, high order harmonics and their sidebands are easily filtered by band-pass filter. When the signal is sampled, the sampling time point is controlled in the program to avoid the switch action moment, which can effectively avoid the voltage spike. Therefore, the switching action mainly affects the fundamental wave $g_{s}(t)$ of the switch, and the expression is shown in

$$
g_{s}(t)=A_{s} \cos \left(2 \pi f_{s} t+\varphi_{s}\right)
$$

Where, $A_{s}$ is the fundamental amplitude, $f_{s}$ and $\varphi_{s}$ is the switching frequency and initial phase of the converter respectively. When $f_{s}$ is an integral multiple of the modulation carrier frequency $f_{c}$, the demodulation result is zero, as shown in (8) and (9). The modulation carrier frequency selected in this system is the same as the switching frequency, so the switching fundamental has no effect on the demodulation process.

$$
\begin{aligned}
& x_{l s}=\int_{0}^{T_{b}} g_{s}(t) \cos \left(\omega_{c} t\right) d t=0 \\
& x_{q s}=\int_{0}^{T_{b}} g_{s}(t) \sin \left(\omega_{c} t\right) d t=0
\end{aligned}
$$

The low frequency noise is introduced in proportional integral (PI) closed-loop regulation of the converter. The spectrum analysis is shown in Fig. 15. It is mainly distributed at the low frequency and can be filtered by the band-pass filter. Therefore, the influence of noise caused by closed-loop regulation on communication system can be ignored.

\section{Communication Protocol Design}

The above design and analysis focus on the physical layer of communication, however, to achieve efficient communication between LEDs for intelligent dimming, 


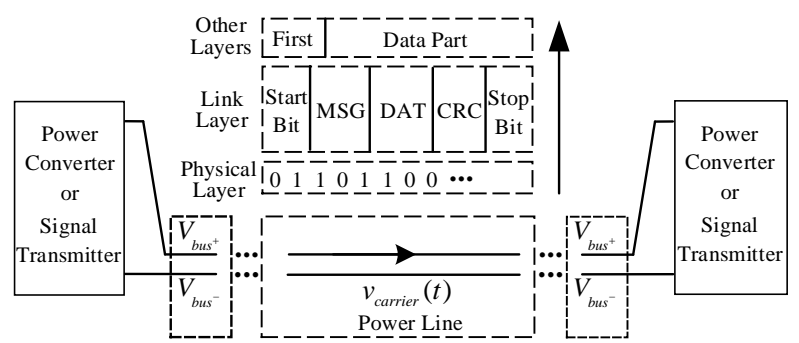

Fig. 12. Communication protocol architecture.

TABLE III. Division of Extended Domain AND Data Domain

\begin{tabular}{|c|c|c|c|c|c|c|c|c|}
\hline 7 & 6 & 5 & 4 & & 3 & 2 & 1 & 0 \\
\hline \multicolumn{2}{|c|}{ Information } & \multicolumn{2}{|c|}{ Data type } & \multicolumn{5}{|c|}{ Acceptance address } \\
\hline \multicolumn{9}{|c|}{ Extended domain MSG } \\
\hline 8 & 7 & 6 & 5 & 4 & 3 & 2 & 1 & 0 \\
\hline \multicolumn{9}{|c|}{ Data } \\
\hline \multicolumn{9}{|c|}{ Data domain DAT } \\
\hline
\end{tabular}

specific communication protocol should be designed. The communication protocol specifies the standards that information exchange must follow in the dimming system, and ensures that the data signals sent by the transmitting equipment can be accurately and smoothly recognized by the receiving equipment. In the design of communication network system, five layer communication protocol structure is adopted, namely physical layer, data link layer, network layer, transportation layer and application layer. This paper mainly studies the physical layer and data link layer which are closely related to power transformation and data transmission. The upper layers protocols are mature enough and will not be discussed in this paper.

The physical layer provides data path and transmits data for signal receiving and transmitting equipment. In the system, the physical layer uses power converter as signal receiving and transmitting equipment, and power line as transmission medium for signal transmission, and the block diagram is shown in Fig. 12.

The data link layer is used to combine the data of the physical layer into data frames according to a certain format, which is responsible for error detection and error recovery of data frames, so as to realize the accurate transmission of data frames. The architecture is shown in Fig. 12. The data frame is the protocol data unit of the data link layer, which needs to define the frame format. This paper uses a custom data frame format: each data frame is 20 bits, in which the first and last bits are the start bit and stop bit respectively. The middle data part includes 8-bit extended field, 9-bit data field and 1-bit CRC check bit. The division of extended domain and data domain is shown in Table III. Extended domain is used to store information type, data type and receiving address. Data domain stores specific data transferred.

Information type represents frame type, including broadcast frame, command frame, status frame and reply frame. The command frame is the frame that the specified host sends the control command to the cluster, the status frame is the frame that the host sends to the cluster to request it to return the current state, and the reply frame is the frame that the specific cluster sends after receiving the command frame or the status frame from the host. Data type is the kind of information stored in data field, including voltage, current, power and other variables. The receiving address is the receiver address of the frame, which can be determined according to the communication equipment in the system. The definition of data frame can be extended according to the actual needs.

\section{Simulation VerificATION}

A power/signal multiplexing modulation system based on boost converter is built in PSIM simulation platform. The block diagram of simulation principle is shown in Fig. 3.

Fig. 13 shows the communication waveform of only one device on the power supply bus, where V1 is the gate signal waveform of the switch in the boost circuit; V2 is the transmitted serial quaternary data signal waveform; V3 is the signal waveform of bus ripple after data demodulation. The period of a single data symbol is $60 \mu \mathrm{s}$. The time delay of receiving and demodulating data signal is $60 \mu \mathrm{s}$. The delay is caused by the demodulation algorithm and does not affect the accuracy of data signal transmission.

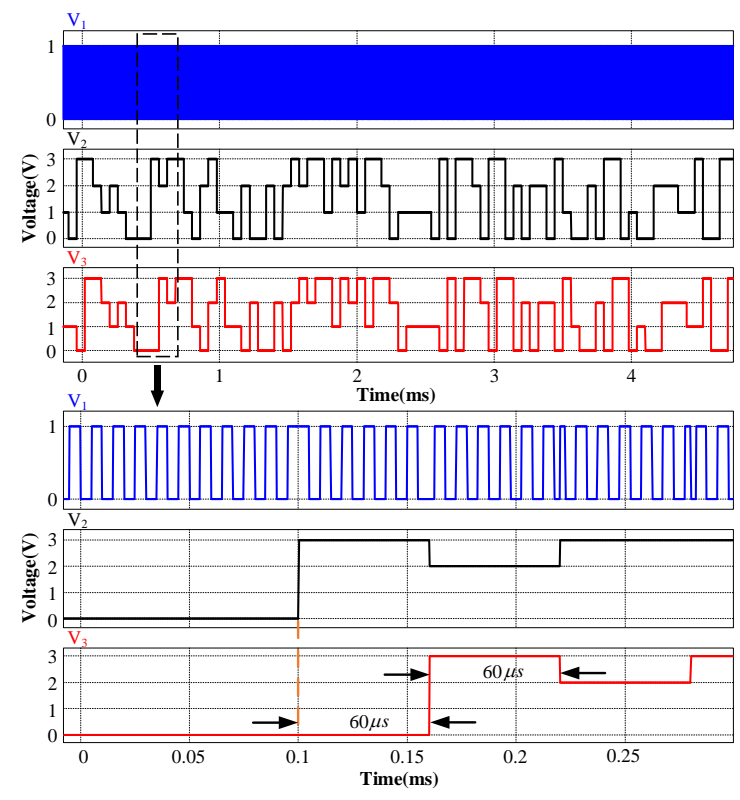

Fig. 13. Waveforms of gate pole signal, transmitting signal and receiving signal of switch tube.

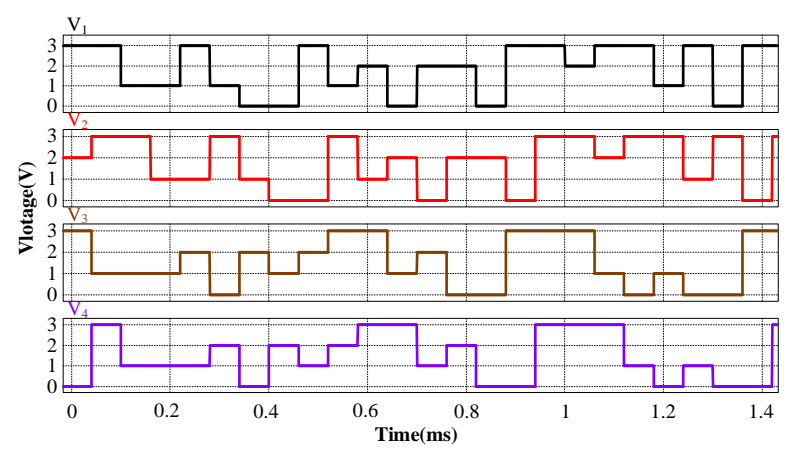

Fig. 14. Waveforms of transmitted signal and received signal. 


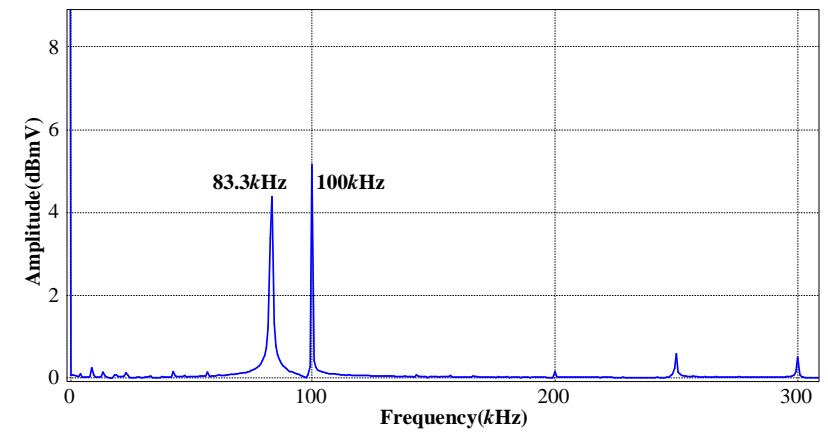

Fig. 15. Bus voltage ripple spectrum of power/signal multiplexing modulation.

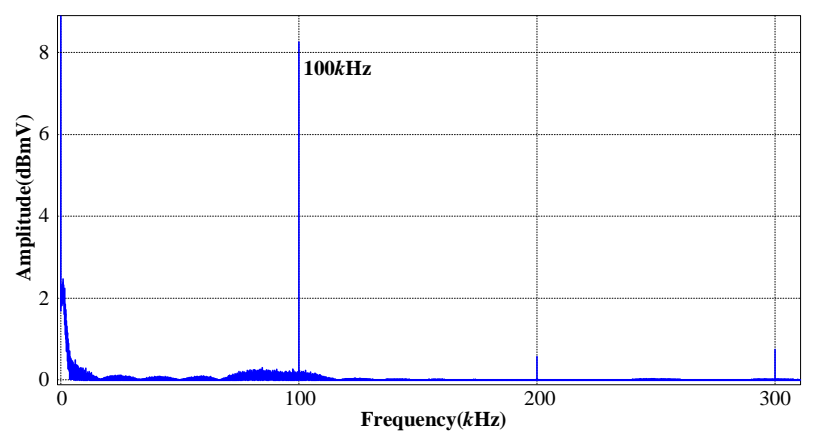

Fig. 16. Bus voltage ripple spectrum of sending random sequence.

When multiple devices are connected to the bus, the device that does not send data adopts the modulation carrier with phase of 0 and frequency of $100 \mathrm{kHz}$ for power modulation. Fig. 14 shows the waveform of power/signal multiplexing modulation and transmission using orthogonal frequency division multiplexing (OFDM) technology when four converters are connected to the bus. Among them, two devices 1 and 2 which transmit data adopt modulation carrier frequencies of $83.3 \mathrm{kHz}$ and $100 \mathrm{kHz}$. Device 3 and 4 are respectively used to receive data signals sent by device 1 and device 2. V1 and V3 are the serial quaternary data signal waveforms sent by device 1 and device 2 respectively; V2 and $\mathrm{V} 4$ are the signal waveforms after data demodulation on the bus. As can be seen from Fig. 14, the OFDM technology can improve the communication efficiency without affecting the accuracy of data transmission.

The frequency spectrum of bus voltage ripple modulated by different carriers is analyzed. When the data signal sent by device 1 and device 2 is "0000 …000", the spectrum result is shown in Fig. 15. It can be found that because the frequency of modulation carrier adopted by device 1 and device 2 is $83.3 \mathrm{kHz}$ and $100 \mathrm{kHz}$ respectively, there is a peak at $83.3 \mathrm{kHz}$ and $100 \mathrm{kHz}$ respectively. When the transmitted data signal is other quaternary random sequence, the result of bus voltage ripple spectrum is as shown in Fig. 16. Because the carrier frequencies of the two transmitting devices are $83.3 \mathrm{kHz}$ and $100 \mathrm{kHz}$ respectively, and the transmitted data signal is a random sequence, there is no peak at $83.3 \mathrm{kHz}$ and $100 \mathrm{kHz}$; The modulation carrier frequency of the two receiving devices is $100 \mathrm{kHz}$, so there is a peak at the frequency of $100 \mathrm{kHz}$, and it also contains high-order harmonic components; The data modulation carrier period is $12 \mu s$ and $10 \mu s$ respectively, and the transmitted data signal symbol period is $60 \mu \mathrm{s}$. The two
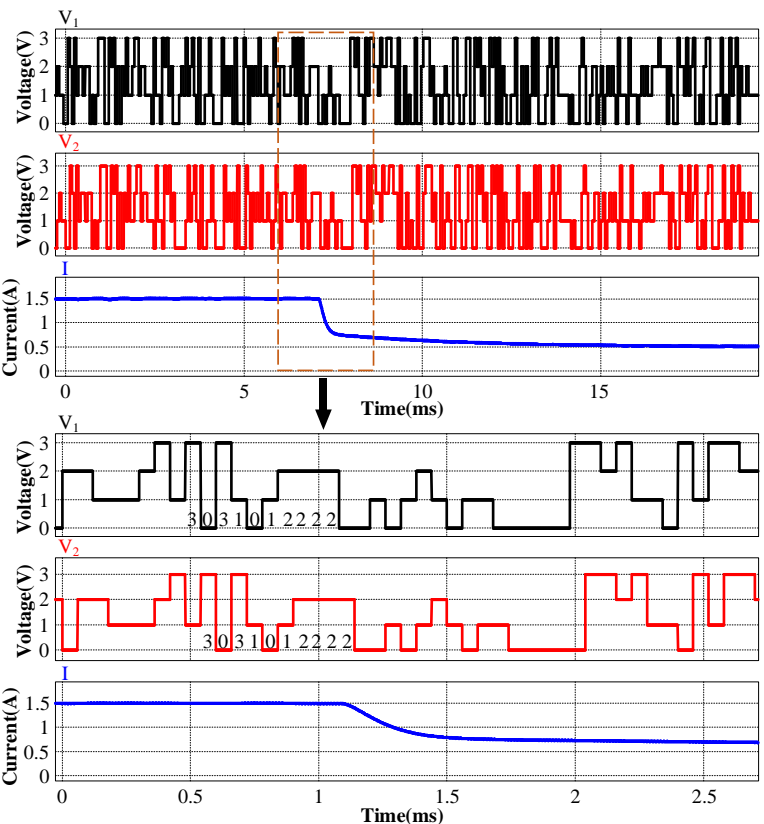

Fig. 17. Power line communication waveforms: reduce illumination command.
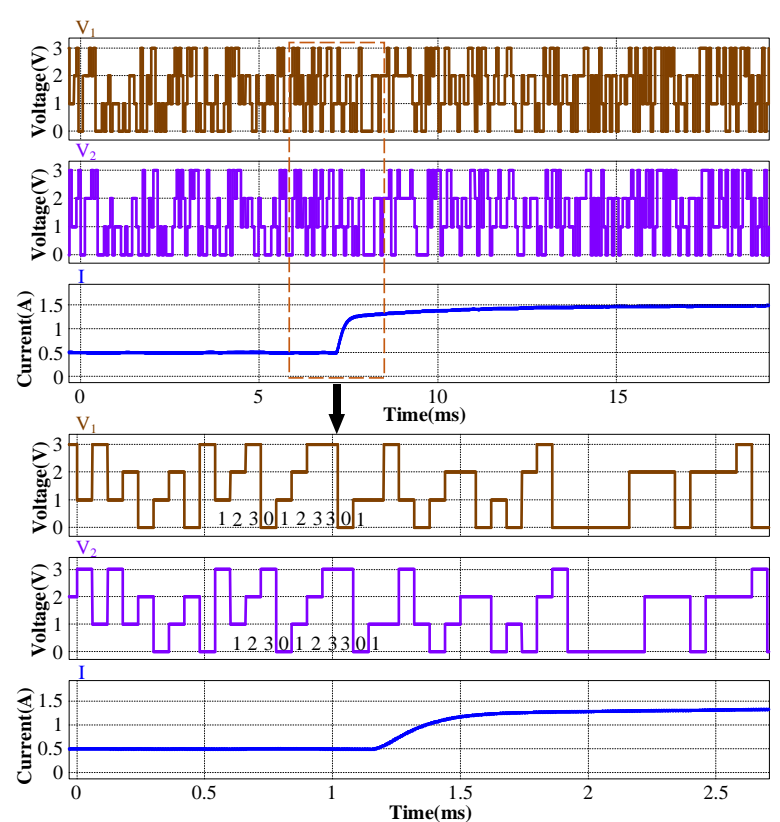

Fig. 18. Power line communication waveforms: add illumination command.

carriers are orthogonal in the symbol period, so the signal can be demodulated, which is in line with the theoretical analysis.

Fig. 17 shows the waveform of the communication signal and the current flowing through the LED when the device sends the light reduction command. Where V1 is the data signal transmitted by the device 1 ; V2 is the data signal received by the device 3 from the bus; $I$ is the current on the LED in device 3. It can be seen that the device 1 sends the light reduction command signal "3031012222", and the device 3 can stably reduce the led to the specified brightness within $10 \mathrm{~ms}$ after receiving the signal. The waveform of light addition command signal is shown in Fig. 18. The above results show that the system can realize intelligent dimming 
control of LED lighting system without additional communication lines.

\section{CONCLUSION}

An intelligent LED dimming system based on switch ripple communication is proposed in this paper. Boost converters are employed as LED drivers, and switching ripple communication is used to achieve power conversion and data communication simultaneously. Data transmits along the common power bus among each boost converters. The QDPSK modulation method and OFDM technology are used to further improve the communication performance. Compared with traditional communication strategy used for intelligent dimming in LED lighting systems, the proposed method does not require additional data transmitters or communication wires, thus the communication has the same reliability with the original LED lighting systems. Besides, the volume, cost and complexity of the system is greatly decreased.

\section{ACKNOWLEDGMENT}

This work is supported by Jiangsu Province Foundation BK20210568 and 20KJB470021, and Jiangsu Innovation and Entrepreneurship Talent Program.

\section{REFERENCES}

[1] P. T. Daely, H. T. Reda, G. B. Satrya, J. W. Kim and S. Y. Shin, "Design of smart LED streetlight system for smart city with web-based management system," in IEEE Sensors Journal, vol. 17, no. 18, pp. 6100-6110, 15 Sep. 2017.

[2] M. Magno, T. Polonelli, L. Benini and E. Popovici, "A low cost, highly scalable wireless sensor network solution to achieve smart LED ligh control for green buildings," in IEEE Sensors Journal, vol. 15, no. 5, pp. 2963-2973, May. 2015.

[3] C. Atıcı, T. Ozcelebi and J. J. Lukkien, "Exploring user-centered intelligent road lighting design: a road map and future research directions," in IEEE Transactions on Consumer Electronics, vol. 57, no. 2, pp. 788-793, May. 2011.

[4] Z. Weng, J. Fang, Z. Weng, G. Zhang and Y. Cheng, "Designed intelligent lighting system of LED based on wireless sensor network technology," 2017 32nd Youth Academic Annual Conference of Chinese Association of Automation (YAC), pp. 398-402, 2017.

[5] W. Lun, K. H. Loo, S. Tan, Y. M. Lai and C. K. Tse, "Bilevel curren driving technique for LEDs," in IEEE Transactions on Power Electronics, vol. 24, no. 12, pp. 2920-2932, Dec. 2009.

[6] S. Tan, "General n-level driving approach for improving electrical-tooptical energy-conversion efficiency of fast-response saturable lighting devices," in IEEE Transactions on Industrial Electronics, vol. 57, no. 4, pp. 1342-1353, Apr. 2010.

[7] K. H. Loo, Y. M. Lai, S. Tan and C. K. Tse, "Stationary and adaptive color-shift reduction methods based on the bilevel driving technique for phosphor-converted white LEDs," in IEEE Transactions on Power Electronics, vol. 26, no. 7, pp. 1943-1953, Jul. 2011.

[8] J. Diaz, E. Rodriguez, L. Hurtado, H. Cacique, N. Vazquez and A Ramirez, "CAN bus embedded system for lighting network applications," 2008 51st Midwest Symposium on Circuits and Systems, pp. 531-534, 2008.

[9] Qing-min Lai, "Remote control system for LED lighting based on CAN bus," 2011 Second International Conference on Mechanic Automation and Control Engineering, pp. 4276-4278, 2011.

[10] S. Matta and S. M. Mahmud, "An intelligent light control system for power saving," IECON 2010 - 36th Annual Conference on IEEE Industrial Electronics Society, pp. 3316-3321, 2010.

[11] F. Leccese, "Remote-control system of high efficiency and intelligent street lighting using a ZigBee network of devices and sensors," in IEEE Transactions on Power Delivery, vol. 28, no. 1, pp. 21-28, Jan. 2013.

[12] L. Hardy and M. Gafen, "A synchronized wireless mesh network model for intelligent lighting control: case study," 2010 Seventh International Conference on Networked Sensing Systems (INSS), pp. 153-156, 2010.

[13] L. Lian and L. Li, "Wireless dimming system for LED street lamp based on ZigBee and GPRS," 2012 3rd International Conference on System Science, Engineering Design and Manufacturing Informatization, pp. 100-102, 2012.

[14] Y. K. Tan, T. P. Huynh and Z. Wang, "Smart personal sensor network control for energy saving in DC grid powered LED lighting system," in IEEE Transactions on Smart Grid, vol. 4, no. 2, pp. 669-676, Jun. 2013.

[15] J. Byun, B. Jeon, J. Noh, Y. Kim and S. Park, "An intelligent selfadjusting sensor for smart home services based on ZigBee communications," in IEEE Transactions on Consumer Electronics, vol. 58, no. 3, pp. 794-802, Aug. 2012.

[16] K. Gill, S. -H. Yang, F. Yao and X. Lu, "A ZigBee-based home automation system," in IEEE Transactions on Consumer Electronics, vol. 55, no. 2, pp. 422-430, May. 2009.

[17] D. Han and J. Lim, "Smart home energy management system using IEEE 802.15.4 and zigbee," in IEEE Transactions on Consumer Electronics, vol. 56, no. 3, pp. 1403-1410, Aug. 2010.

[18] G. Shahzad, H. Yang, A. W. Ahmad and C. Lee, "Energy-efficient intelligent street lighting system using traffic-adaptive control," in IEEE Sensors Journal, vol. 16, no. 13, pp. 5397-5405, Jul. 2016.

[19] S. Shao et al., "Design and analysis of a visible-light-communication enhanced WiFi system," in IEEE/OSA Journal of Optical Communications and Networking, vol. 7, no. 10, pp. 960-973, Oct. 2015 .

[20] K. P. Pujapanda, "LiFi integrated to power-lines for smart illumination cum communication," 2013 International Conference on Communication Systems and Network Technologies, pp. 875-878, 2013.

[21] J. Song, W. Ding, F. Yang, H. Yang, B. Yu and H. Zhang, "An indoor broadband broadcasting system based on PLC and VLC," in IEEE Transactions on Broadcasting, vol. 61, no. 2, pp. 299-308, Jun. 2015.

[22] W. Xiao, L. Bo and R. Kaur, "Design of LED lighting system based on power line communication," 2020 5th International Conference on Computer and Communication Systems (ICCCS), 2020, pp. 835-839.

[23] C. Li, J. Wu and X. He, "Realization of a general LED lighting system based on a novel power line communication technology," 2010 Twenty-Fifth Annual IEEE Applied Power Electronics Conference and Exposition (APEC), pp. 2300-2304, 2010.

[24] J. Wu, J. Du, Z. Lin, Y. Hu, C. Zhao and X. He, "Power conversion and signal transmission integration method based on dual modulation of DC-DC converters," in IEEE Transactions on Industrial Electronics, vol. 62, no. 2, pp. 1291-1300, Feb. 2015 